\title{
Stable Genetic Influence on Anxiety-Related Behaviours Across Middle Childhood
}

\author{
Maciej Trzaskowski • Helena M. S. Zavos • \\ Claire M. A. Haworth • Robert Plomin • Thalia C. Eley
}

Published online: 16 July 2011

(C) The Author(s) 2011. This article is published with open access at Springerlink.com

\begin{abstract}
We examined the aetiology of anxiety symptoms in an unselected population at ages 7 and 9 , a period during which anxiety disorders first begin to develop (mean age at onset is 11 years). Specifically, the aim of the study was to investigate genetic and environmental continuity and change in components of anxiety in middle childhood. Parents of over 3,500 twin pairs completed the AnxietyRelated Behaviours Questionnaire (ARBQ) when twins were 7 and 9 years old. Multivariate-longitudinal analyses were conducted to examine genetic and environmental influences on stability and change in four anxiety scales: Negative Cognition, Negative Affect, Fear and Social Anxiety. We found moderate temporal stability in all four scales from 7 to 9 years (correlations ranging from 0.45 to 0.54 ) and moderate heritability (average 54\%). Both shared and non-shared environmental influences were modest (average 18\%-28\% respectively). Genetic factors (68\%) explained most of the homotypic continuity in anxiety. We show that homotypic continuity of Anxiety-Related Behaviours (i.e. the continuation of one specific type of anxiety over time) was largely driven by genetic factors. In contrast, though more varied, heterotypic continuity between some traits (i.e. the change from one type of anxiety-related behaviour into another over time) was mainly due to shared-environmental factors.
\end{abstract}

M. Trzaskowski $(\bowtie) \cdot$ H. M. S. Zavos $\cdot$ C. M. A. Haworth •

R. Plomin • T. C. Eley

King's College London, MRC Social, Genetic and Developmental

Psychiatry Centre, Institute of Psychiatry,

Box PO80, De Crespigny Park,

London SE5 8AF, UK

e-mail: maciej.trzaskowski@kcl.ac.uk
Keywords Anxiety · Twins · Genetics · Genetic continuity · Developmental anxiety · Longitudinal-multivariate study . Anxiety-related behaviours $\cdot$ Middle childhood

\section{Introduction}

Anxiety is often long lasting and is associated with many negative outcomes, such as poor academic performance, poor social adjustment and poor interpersonal adjustment (Fombonne et al. 2001; Last et al. 1997; Otto et al. 2001). Anxiety disorders have been reported to affect as many as $30 \%$ of individuals during childhood (Costello et al. 2003; Ford et al. 2003), with mean onset estimated around 11 years (Kessler et al. 2005). There is also evidence that sub-clinical anxiety related behaviours (ARBs) are predictive of subsequent anxiety disorders. Specifically, individuals who suffer from high levels of anxiety in childhood are at an increased risk of developing anxiety disorders later in life (Last et al. 1996, 1997; Pine 2007). Studying ARB's just prior to the onset of anxiety disorders could therefore capture the aetiological changes involved in the early stages of the development of anxiety.

Structure and Continuity of Anxiety

Traditionally, studies investigating the phenotypic structure of anxiety in young people have tended to consider the complexity of this phenotype from one of two rather different perspectives. Those working from the clinical perspective have tried to identify clusters of symptoms that reflect those that form each of the anxiety diagnoses. For example, the factor structure of anxiety symptoms reported by both clinical and population-based samples of children 
tend to reflect the structure of anxiety disorders outlined within the DSM-IV (Spence 1997). A contrasting approach is to examine aspects of personality or temperament, such as shyness/behavioural inhibition (Gladstone et al. 2005) or negative affect, which may be associated with many different anxiety disorders (Brady and Kendall 1992; Caspi et al. 1995; Rosenbaum et al. 1993). Of note, negative affect has been described as the common feature of anxiety and depression (Clark and Watson 1991). This type of approach therefore focuses the mind on what is in common between different aspects of anxiety rather than how they differ from one another. In sum, it is unclear from the literature whether anxiety is better described by considering what is in common between different types of symptom and behaviour, or by considering what is distinct to each.

This phenotypic complexity leads to several interesting longitudinal questions. Specifically, to what extent are these constructs stable over time, and are there stronger associations within or between anxiety sub-types across middle childhood? Studies looking at longitudinal patterns have found evidence of both homotypic continuity (prediction of a disorder by the same disorder), and heterotypic continuity (prediction of a disorder by another disorder). For example, a Dutch study of 10 12 year old children demonstrated that symptoms of separation anxiety (SAD), social phobia $(\mathrm{SoPh})$ and panic disorder (PD) were characterised by strong homotypic continuity (Ferdinand et al. 2007), indicating specificity of anxiety constructs. In contrast, symptoms of generalised anxiety disorder (GAD) were characterised by stronger heterotypic continuity. Homotypic continuity of anxiety has also been demonstrated in a longitudinal study of participants from 11 to 32 years old (Gregory et al. 2007). Adults with anxiety disorders were more likely to have experienced anxiety in adolescence than any other disorder. This pattern was particularly pronounced for phobias, with specific phobias in adulthood being preceded by juvenile phobias of the same type. Given this mixed pattern of both homotypic and heterotypic continuity in the anxiety domain, it is pertinent to ask how genetic and environmental factors account for stability and change in these phenotypes and their associations over time.

\section{Genetic and Environmental Influences}

Behavioural genetic studies of anxiety in children indicate a general pattern of moderate genetic influence. However, the heritability has been shown to vary depending on age, gender and on whether anxiety is treated as a clinical disorder or as an aspect of temperament, such as shyness (Feigon et al. 2001; Gregory and Eley 2007).

The literature on sources of stability and change over time are more consistent. As with depression, it would appear that genetic influence is more general, i.e., common across the anxiety sub-types and across-time, whereas environmental influences appear to be more scale and time-specific, thus accounting for specificity and change of symptoms (Eaves and Silberg 2008; Kendler et al. 2008a, b, c; Lau and Eley 2006). One of the most informative of this group of studies included repeated assessments of fear symptoms in a cohort of twins aged 8-9 at wave 1 and 19-20 by wave 4 (Kendler et al. 2008b). The tight age-range within the cohort makes this dataset particularly valuable. Results indicated genetic continuity across middle childhood, although new genetic influences also emerged throughout adolescence and adulthood. Of note, the genetic factor influencing continuity attenuated over time, with new genetic influences coming on board during adolescence, suggesting the effects of early genetic factors acting on fear diminish over time. Other studies have also suggested genetic influence on continuity. For example, in the measure of behavioural inhibition in infancy (Robinson et al. 1992) and of mixed anxiety and depression in middle childhood (Kendler et al. 2008c) genetic factors were reported to substantially contribute to continuity and change. This would suggest the maintenance of anxiety sub-types across childhood to be most likely driven by genetic rather than environmental factors.

\section{Current Study}

Here we present a longitudinal analysis of four anxietyrelated scales (negative cognition, negative affect, fear and social anxiety) during middle childhood. These resulted from factor analyses (reported elsewhere; Hallett et al. 2009), performed on data drawn from parent ratings on the Anxiety-Related Behaviours Questionnaire (ARBQ). This measure was favoured because it was composed of items that were selected to reflect both a clinical-diagnostic approach to anxiety and relevant aspects of temperament/personality. Given that traditional approach tended to focus on each aspect separately (clinical symptoms versus temperament), it could be argued that combining both approaches under the one measure could capture more successfully relationship between all these aspects. We chose to examine anxietyrelated behaviours in representative population-based sample in middle childhood as it directly precedes the mean age of onset of anxiety disorders (11 years, Kessler et al. 2005) and thus it has a potential to tap in to the prodromal period of anxiety. However, given possibility that some of our participants could have already had anxiety at the time the data was collected, it is likely that we were tapping into combination of prodromal as well as already existing early stages of anxiety. 
Previous analysis of the same sample explored the aetiology of the four scales within each age ( 7 and 9 years). The results showed that at each age, genes indicated mainly a general predisposition to anxiety, whereas environments contributed largely to the differentiation between the anxiety scales (Hallett et al. 2009). Of note, the influence of shared environment was markedly higher for fear and negative affect at 7 and 9 and multivariate analysis showed strong shared-environmental correlation between these two traits. The current study sought to further our understanding of this overlap by extending the multivariate design longitudinally to analyse the aetiology of continuity and change across the four anxiety scales during middle childhood. Specifically we asked three main questions.

First, we sought to investigate the stability of anxiety traits during middle childhood. This question concerned the strength of the phenotypic longitudinal correlations. Given evidence of stability of various measures of anxiety (Ferdinand et al. 2007; Gregory et al. 2007; Kendler et al. 2008b) and that previous factor analyses using this sample revealed the same phenotypic structure at both ages (Hallett et al. 2009), we predicted moderate phenotypic stability across this period. Second, the generalist genes hypothesis suggests that the influences of genes tends to be broader, acting across times and phenotypes with environmental influences more time specific and phenotype specific (Eley 1997; Plomin and Kovas 2005). Numerous studies also indicate that genetic factors tend to account for the majority of stability in anxiety-related traits (e.g. Kendler et al. 2008b). We therefore expected strong genetic stability in all our scales (referred to here as homotypic continuity). Finally, the extent to which genetic influences accounted for continuity across traits (referred to here as heterotypic continuity) was investigated. For example, we examined the extent to which genetic influences account for the association between negative cognition at age 7 and negative affect at age 9. The aetiological processes acting on these traits across middle childhood have not yet been explored, but we expect genetic influences on heterotypic continuity to be significant though lower than genetic homotypic continuity.

\section{Methods}

Sample

Participants were drawn from the Twins Early Development Study (TEDS) which is an ongoing longitudinal study following twins born in England and Wales between the years 1994 and 1996 (Oliver and Plomin 2007). The TEDS sample has been shown to be reasonably representative of the population (for further details see: Kovas et al. 2007).
TEDS research was approved by the Institute of Psychiatry Ethics Committee, and all participants gave informed consent. After medical exclusions, the sample consisted of 7,834 twin pairs at age 7 and 3,644 pairs at age 9 . The sample for the 9-year study was restricted to just two out of three cohorts resulting in reduced sample size. Zygosity of twins was determined using a combination of parental rating of similarity and DNA genotyping (Price et al. 2000).

\section{Measures}

Anxiety measures were extracted from the parent-rated Anxiety-Related Behaviours Questionnaire (ARBQ) first described by Eley et al. (2003). This measure includes items reflecting symptoms of anxiety disorder and aspects of anxiety related temperament. In addition, previously reported statistics have shown that the items have good construct validity and adequate internal consistency (Cronbach's $\alpha 0.64$ at 7 and 0.66 at 9) (Hallett et al. 2009). Previous factor analysis revealed five scales: negative cognition, negative affect, fear, social anxiety and obsessive compulsive behaviour (OCB) (detailed information of item loadings can be found in Hallett et al. 2009). Of note, the measure serves to assess individual differences in anxiety ratings within the general population. It does not constitute a diagnostic tool for internalizing disorders, but shows some similarities to screening questionnaires such as the Screen for Child AnxietyRelated Emotional Disorders (Birmaher et al. 1997) and the Spence Children's Anxiety Scale (Spence et al. 2003). Parent-ratings were largely contributed by mothers who accounted for $98 \%$ and $99 \%$ of returned data at ages 7 and 9 respectively. The OCB scale showed a categorical distribution and the items that loaded onto this scale did not appear to give a comprehensive representation of OCB symptoms. Consequently, this subscale was excluded from the analyses.

\section{Twin Design}

Quantitative genetic analyses were used to estimate additive genetic (A), shared environmental (C) and non-shared environmental (E) influences. Additive genetic refers to the effects of alleles, which cumulatively add up to the main effect. Shared environments refer to environmental effects that make two children in the family more similar, whereas non-shared (unique) environments are those that lead to differences between family members. This approach takes advantage of the two types of twins: monozygotic (MZ) twins who are genetically 100\% identical, and dizygotic (DZ) twins who share on average $50 \%$ of their segregating alleles. Relative differences in within-pair correlations are then used to estimate A, C and E. Where 
MZ correlations are higher than DZ twins, genetic influence (A) is assumed to play a role. Any effects that contribute to within-pair similarities but are not genetic, are attributed to shared environmental influences (C) and the extent to which MZ twins differ is attributed to non-shared environmental influences (E). Detailed description of this model and the discussion of related issues can be found elsewhere (Plomin et al. 2008).

\section{Selecting Models of Best Fit}

Models were fitted using raw data maximum likelihood. The fit statistics provided by Mx (Neale 1997) for raw data modelling is minus twice the log likelihood ( $-2 L L)$ of the observations. This estimate is not an overall measure of fit, but provides a relative measure of fit, since differences in $-2 \mathrm{LL}$ between models are distributed as $\chi^{2}$. Therefore, to examine the overall fit of the genetic model it is necessary to compare the $-2 \mathrm{LL}$ to that of a saturated model. Consistent with the principle of parsimony, the fit of sub-models was assessed by $\chi^{2}$ difference tests and the Akaike's Information Criterion (AIC $=\chi^{2}-2 \mathrm{df}$ ) with lower $\chi^{2}$ values and more negative AIC values indicating a better fit (Neale and Cardon 1992). Confidence intervals of parameter estimates were obtained by maximum likelihood. Model-fitting analyses can also test for sex differences in the patterns of aetiological factors by comparing models, which vary in their assumptions and specifications of the genetic and environmental parameters in males and females.

\section{Multivariate and Longitudinal Models}

Complex developmental disorder can have widely varying aetiological structures, shape of which will have a strong theoretical implication on the understanding of its nosology. Multivariate longitudinal relationships can, for example, have an underlying trait-like construct that is stable across time, or have correlated aetiological components that explain only some proportion of bi-variate relationships. Drawing on previous findings we fitted three most plausible models to determine the best aetiological structure underlying our data: a common factor model, an independent pathway model and a correlated factors model (see Fig. 1). The first most restricted model was the common factor model. This model assumes that there is a single latent factor underlying all eight variables (four at year 7 and four at year 9). Variance of that latent factor is then decomposed into genetic and environmental influences. In that, these influences are equal for all measures that make up this composite. Any remaining variance (not explained by the latent factor) is than calculated as trait specific genetic and environmental influences.
Model 1: Common Pathway Model
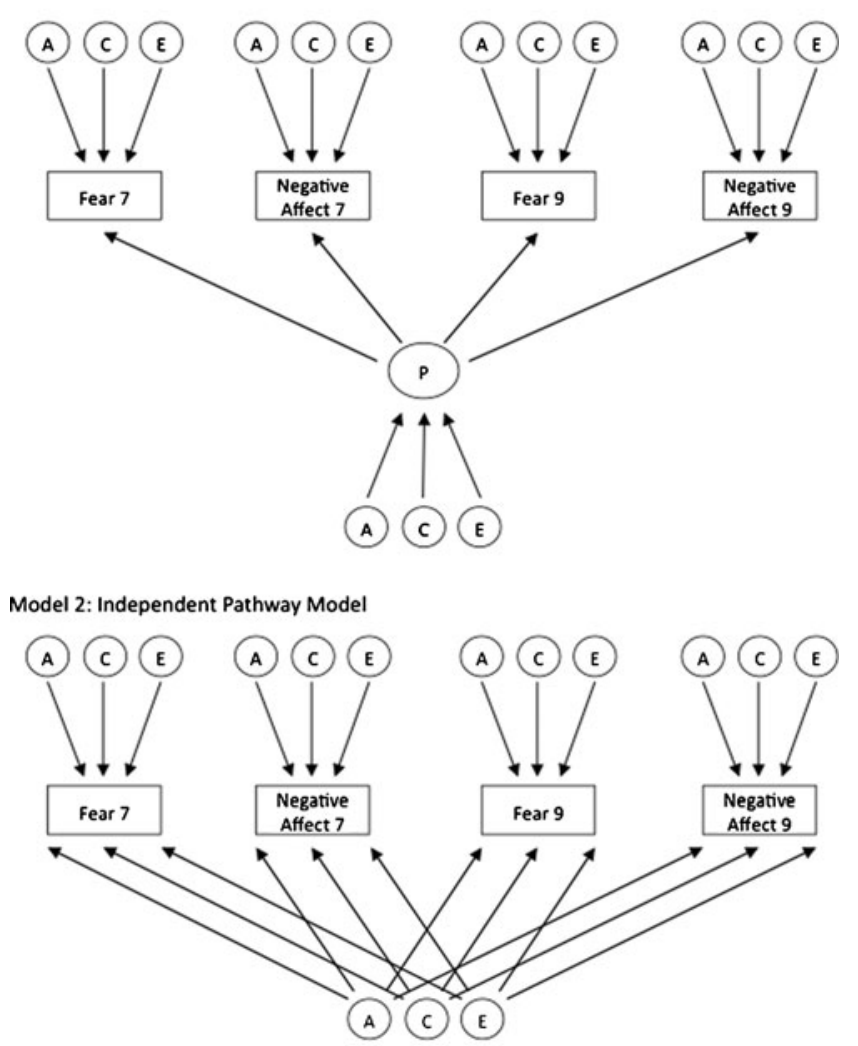

Model 3: Correlated Factors Model

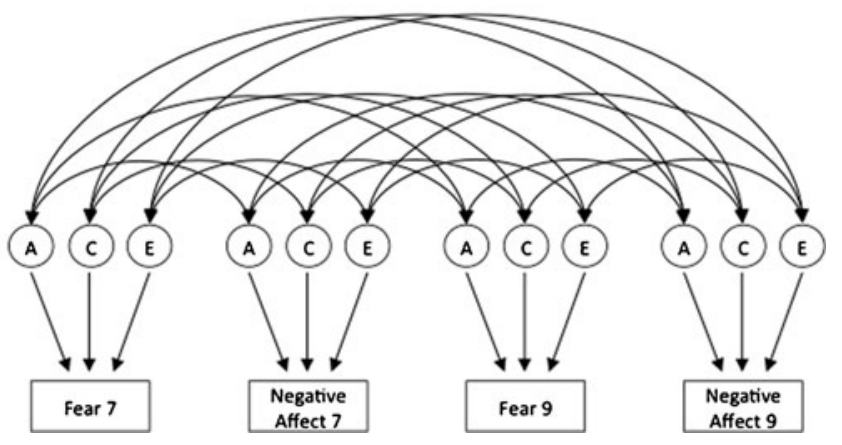

Fig. 1 Illustrative example of common pathway, independent pathway and correlated factors models of Fear at 7 and 9 and Negative Affect at 7 and 9. Full model contained 4 variables: 2 illustrated here plus Social Anxiety, and Negative Cognition at both ages

The second, less restricted model, was the independent pathways model. A common phenotypic factor is not assumed in this model, instead there is one set of common $\mathrm{A}, \mathrm{C}$ and $\mathrm{E}$ terms directly influencing each of the measured variables. The influence each of the general factors has on the specific variables is free to differ for each variable (i.e., the sizes of the influences are independent of one another). There are also specific A, C and E residual terms on each of the eight variables. This model tests the hypothesis that there are common genetic and environmental factors that 
influence all eight variables, accounting for their correlation, in addition to variable specific-factors.

The final and least restricted model was the correlated factors model, which includes A, C and E terms for each of the variables, and each of the sets of terms is allowed to correlate with one another. That is, all eight variables could correlate via a genetic route (A) or due to environmental influences ( $\mathrm{C}$ or $\mathrm{E})$. This model tests the hypothesis that the correlation amongst the eight variables is due to correlations amongst the individual sets of latent factors that influence each of them. The correlated factors model incorporated different parameter estimates for each scale with correlations between the genetic $(\mathrm{rG})$, non-shared $(\mathrm{rE})$, and shared (rC) environmental influences (see Fig. 1).

The independent pathway model and common factor model denote a more restricted representation of the data, testing the hypothesis that there are common genetic and environmental influences underlying all subscales, whereas correlated factors model makes no such assumptions (for further explanation see Neale and Cardon 1992). We chose these models for two reasons. One, genetic and environmental changes across time can have very different patterns to those observed within a single time point. Thus, findings from previously published cross-sectional study (Hallett et al. 2009) were suggestive but not definitive to our study. Two, neither the cross-sectional nor the longitudinal analyses indicate correlational patterns that would suggest structures such as multiple common factors. Thus, following the rule of parsimony we tested a single common factor.

\section{Results}

Longitudinal Phenotypic Associations

Within-variable and between variable phenotypic correlations over time are shown in Table 1. As expected, within-scale phenotypic correlations over time (on diagonal values in bold), were moderate varying from 0.45 for negative affect to 0.54 for social anxiety. Between-scale phenotypic correlations over time (off diagonal), were somewhat weaker ranging from 0.17 (social anxiety age 7-negative affect age 9) to 0.32 (negative affect age 7-negative cognition age 9).

\section{Model Fitting}

Table 2 summarises previously published twin correlations for all 4 subscales at ages 7 and 9 (Hallett et al. 2009). The results did not show a dominance effect, thus all further analyses were performed using ACE models. The results also showed neither qualitative nor quantitative sex differences; therefore all calculations were performed on a data separated by zygosity only. Saturated model also did not indicate §mean nor variance differences allowing them to be equated across twins and across zygosities. Fit statistics from the multivariate longitudinal models are presented in Table 3. The AIC was the preferred index for model selection as this takes into account the large sample size, which can inflate even small differences between the observed and expected variance/covariance structure. A low negative AIC value indicates good fit for the correlated factors model. In contrast, high positive AIC value for both the common and independent pathway models indicate that these models do not fit the data as well. Consequently, the correlated factors model was selected as the model that best explained our data.

\section{Genetic and Environmental Influences on Homotypic Continuity}

The level of genetic homotypic continuity within variables is shown in the top third section of Table 4 (on diagonal bold font values). Findings indicate moderate to high genetic continuity for all variables, with social anxiety showing the strongest genetic correlation over time (0.66) and negative affect the lowest (0.55). The proportion of longitudinal co-variance explained by genetic factors (top third section of Table 5, on diagonal bold font values) was high for all scales, ranging from $57 \%$ for negative affect to $67 \%$ for social anxiety. This means that $57 \%$ of the continuity between 7 and 9 years in negative affect, is explained by stable genetic factors.

Table 1 Phenotypic correlations between anxiety measures at age 7 and at age 9

\begin{tabular}{|c|c|c|c|c|}
\hline & Negative Cognition 9 & Negative Affect 9 & Fear 9 & Social Anxiety 9 \\
\hline Negative Cognition 7 & $0.50(0.48-0.51)$ & $0.30(0.27-0.30)$ & $0.25(0.24-0.27)$ & $0.27(0.26-0.29)$ \\
\hline Negative Affect 7 & $0.32(0.30-0.32)$ & $0.45(0.43-0.48)$ & $0.25(0.23-0.27)$ & $0.24(0.22-0.24)$ \\
\hline Fear 7 & $0.21(0.21-0.23)$ & $0.19(0.19-0.23)$ & $0.52(0.52-0.54)$ & $0.26(0.25-0.28)$ \\
\hline Social Anxiety 7 & $0.23(0.22-0.27)$ & $0.17(0.17 .19)$ & $0.25(0.23-0.28)$ & $0.54(0.54-0.56)$ \\
\hline
\end{tabular}

The values on the diagonal (in bold) represent homotypic continuity (i.e. same variable across time). The values on the off diagonal represent heterotypic continuity (i.e. different variables across time). $95 \%$ confidence intervals are in parentheses 
Table 2 Pearson's twin correlations at age 7 and 9

For cross-twin-cross-trait correlation please see Hallett et al. (2009)

\begin{tabular}{llllll}
\hline Age & 7 & & 9 & \\
\cline { 2 - 3 } \cline { 5 - 6 } Zygosity & $\mathrm{MZ}$ & $\mathrm{DZ}$ & $\mathrm{MZ}$ & $\mathrm{DZ}$ \\
\hline Negative Cognition & $0.60(0.58-0.62)$ & $0.35(0.32-0.37)$ & & $0.69(0.66-0.71)$ & $0.40(0.37-0.44)$ \\
Negative Affect & $0.71(0.69-0.72)$ & $0.45(0.43-0.48)$ & & $0.69(0.66-0.72)$ & $0.47(0.43-0.50)$ \\
Fear & $0.76(0.75-0.77)$ & $0.46(0.44-0.49)$ & & $0.78(0.76-0.80)$ & $0.49(0.46-0.52)$ \\
Social Anxiety & $0.70(0.68-0.72)$ & $0.31(0.28-0.35)$ & & $0.77(0.75-0.59)$ & $0.48(0.45-0.51)$ \\
\hline
\end{tabular}

The stability (cross-time correlation) of shared environmental influences, values (in bold) on diagonal of the middle section of Table 4, was moderate (0.35-0.63). Negative cognition correlated the lowest over time 0.35 . The proportion of variance explained by shared environment was significantly lower than that explained by genetic factors, accounting for between $8 \%$ (negative cognition) and $28 \%$ (negative affect) of continuity across time (see middle section of Table 5, values on diagonal).

Non-shared environment showed strong age specificity across all anxiety scales, with correlations over time ranging from just 0.24 (negative affect) to 0.42 (social anxiety; see bottom section of Table 4 , values on diagonal). The proportion of within-variable continuity explained by non-shared environment was similar to shared environment, varying from $13 \%$ for negative affect to $26 \%$ for negative cognition (see bottom third section of Table 5, values on diagonal).

Genetic and Environmental Influences on Heterotypic Continuity

Although heterotypic genetic continuity was markedly lower and more varied than homotypic genetic continuity (0.11-0.41; see top section of Table 4, values off diagonal), there were several exceptions. Most notably, the relationship between early negative cognition and later negative affect explained $66 \%$ of the covariance with genetic correlation estimated at 0.41 .

Shared environmental influences were found to show substantial continuity over time across most scales. The highest shared environmental correlation was between early social anxiety and later fear (shared environmental correla- tion $=0.81$; middle section of Table 4 , values off diagonal). The proportions of covariance over time due to shared environment were moderate to high, with that for the association between early negative cognition and later affect being the lowest (21\%) and that for early negative affect and later fear being the highest $(62 \%$; see middle third of Table 5, values off diagonal). Non-shared environment was age and scale specific; non-shared environmental correlations were low $(0.09-0.23)$ and therefore explained little of the covariance over time $(4 \%-28 \%$; see bottom third of Table 5, values off diagonal).

It is important to mention that the overlapping confidence intervals of the current results suggested that most of the observed differences between the estimates were not significant.

\section{Discussion}

We extended previous work by showing that diverse aspects of anxiety showed strong stability over time. Moreover, this stability was largely due to continuity of genetic influences whereas change resulted mainly from novel environmental influences, which was in line with previous findings (Kendler et al. 2008b). These findings are interpreted with respect to the "General genes" hypothesis (Eley 1997; Plomin and Kovas 2005) which suggests that the influence of genes tend to be broad, acting across times and phenotypes with environmental influences more timespecific and phenotype-specific. Cross-trait (heterotypic) stability showed greater diversity of genetic and shared environmental effects. Importantly however, although it was predominantly the influence of genes that tended to be

Table 3 Multivariate longitudinal fit statistics across middle childhood

\begin{tabular}{llllrr}
\hline Model & -2LL & df & $\chi^{2}$ & $\Delta$ df & \multicolumn{1}{c}{$p$} \\
\hline Saturated & 203767.10 & 82,929 & - & - & - \\
Correlated factor solution & 204078.86 & 83,117 & 311.76 & 188 & 0.001 \\
Independent pathway & $206,580.35$ & 83,177 & 2813.25 & 248 & 0.001 \\
Common pathway & $207,777.34$ & 83,191 & 4010.24 & 262 & 0.001 \\
\hline
\end{tabular}

$(-2 \mathrm{LL})$ - twice difference in $\log$ likelihood between null and alternative models, (df) - degrees of freedom, $\left(\chi^{2}\right)$ - chi square, (p) - $p$ value, (AIC) - Akaike's Information Criterion, $\left(\Delta \chi^{2}\right)$ - between-model difference in chi-square, $(\Delta \mathrm{df})$ - between-model difference in degrees of freedom 
Table 4 Genetic and environmental correlations between anxiety-related behaviours at 7 and 9 years

\begin{tabular}{|c|c|c|c|c|c|}
\hline & & Negative Cognition 9 & Negative Affect 9 & Fear 9 & Social Anxiety 9 \\
\hline \multirow[t]{4}{*}{ A } & Negative Cognition 7 & $0.63(0.53-0.63)$ & $0.41(0.30-0.49)$ & $0.23(0.14-0.26)$ & $0.24(0.14-0.31)$ \\
\hline & Negative Affect 7 & $0.32(0.22-0.36)$ & $0.55(0.44-0.56)$ & $0.16(0.06-0.23)$ & $0.19(0.08-0.20)$ \\
\hline & Fear 7 & $0.25(0.16-0.28)$ & $0.11(0.02-0.16)$ & $0.60(0.53-0.67)$ & $0.25(0.16-0.25)$ \\
\hline & Social Anxiety 7 & $0.21(0.13-0.21)$ & $0.11(0.02-0.16)$ & $0.21(0.14-0.21)$ & $0.66(0.59-0.66)$ \\
\hline \multirow[t]{4}{*}{$\mathrm{C}$} & Negative Cognition 7 & $0.35(0.01-0.51)$ & $0.39(0.07-0.67)$ & $0.72(0.40-0.83)$ & $0.61(0.33-0.61)$ \\
\hline & Negative Affect 7 & $0.59(0.34-0.82)$ & $0.53(0.35-0.68)$ & $0.74(0.51-0.94)$ & $0.52(0.34-0.55)$ \\
\hline & Fear 7 & $0.41(0.12-0.56)$ & $0.59(0.34-0.78)$ & $0.63(0.43-0.76)$ & $0.52(0.30-0.74)$ \\
\hline & Social Anxiety 7 & $0.51(0.25-0.71)$ & $0.69(0.48-0.82)$ & $0.81(0.61-0.96)$ & $0.55(0.35-0.75)$ \\
\hline \multirow[t]{4}{*}{$\mathrm{E}$} & Negative Cognition 7 & $0.38(0.32-0.42)$ & $0.12(0.05-0.12)$ & $0.14(0.08-0.15)$ & $0.23(0.17-0.24)$ \\
\hline & Negative Affect 7 & $0.17(0.11-0.20)$ & $0.24(0.18-0.24)$ & $0.04(0.02-0.04)$ & $0.11(0.05-0.13)$ \\
\hline & Fear 7 & $0.09(0.03-0.12)$ & $0.11(0.04-0.11)$ & $0.31(0.25-0.32)$ & $0.14(0.08-0.20)$ \\
\hline & Social Anxiey 7 & $0.22(0.17-0.22)$ & $0.10(0.04-0.10)$ & $0.12(0.06-0.13)$ & $0.42(0.37-0.42)$ \\
\hline
\end{tabular}

The values in bold on the diagonal represent homotypic continuity attributable to additive genetics (A), shared environment (C) and to non-shared environment (E). The values on the off diagonal values represent heterotypic continuity for additive genetics (A), shared environment (C), and non-shared environment $(\mathrm{E})$

broader, shared environment was significant for several heterotypic relationships, specifically fear and negative affect.

\section{Implications}

The current findings have several implications for understanding the aetiological processes involved in anxiety during middle childhood. First, the fact that genes have a general influence across time is useful for informing future quantitative and molecular research. For example, substantial genetic continuity provides a rationale for combining wider age ranges within middle childhood, which could be useful for combining samples to improve power for molecular genetic analyses. Furthermore, the results from homotypic and heterotypic continuity could be used when designing genome-wide association studies (GWAS). For example, high homotypic continuity gives a good rationale for designing a longitudinal GWAS for each scale. Heterotypic patterns, on the other hand, were significantly lower and more varied. This suggested some scale specificity, highlighting that these scales could not be entirely explained by a single genetic factor. In consequence, this suggested that multivariate GWAS designs would hold very little hope for finding genetic variance common to all scales. The exception to that trend was the

Table 5 Proportion of covariance explained between anxiety-related behaviours at 7 and 9 years

\begin{tabular}{|c|c|c|c|c|c|}
\hline & & Negative Cognition 9 & Negative Affect 9 & Fear 9 & Social Anxiety 9 \\
\hline \multirow[t]{4}{*}{ A } & Negative Cognition 7 & $0.66(0.54-0.76)$ & $0.66(0.47-0.78)$ & $0.47(0.27-0.54)$ & $0.44(0.25-0.63)$ \\
\hline & Negative Affect 7 & $0.52(0.35-0.65)$ & $0.57(0.44-0.69)$ & $0.35(0.14-0.55)$ & $0.40(0.17-0.40)$ \\
\hline & Fear 7 & $0.63(0.39-0.64)$ & $0.28(0.04-0.55)$ & $0.66(0.56-0.67)$ & $0.51(0.32-0.57)$ \\
\hline & Social Anxiety 7 & $0.49(0.31-0.56)$ & $0.31(0.06-0.54)$ & $0.48(0.32-0.56)$ & $0.67(0.58-0.70)$ \\
\hline \multirow[t]{4}{*}{$\mathrm{C}$} & Negative Cognition 7 & $0.08(0.00-0.18)$ & $0.21(0.04-0.34)$ & $0.38(0.21-0.43)$ & $0.32(0.16-0.46)$ \\
\hline & Negative Affect 7 & $0.32(0.17-0.47)$ & $0.28(0.17-0.30)$ & $0.62(0.44-0.80)$ & $0.48(0.31-0.57)$ \\
\hline & Fear 7 & $0.26(0.08-0.47)$ & $0.58(0.34-0.79)$ & $0.21(0.13-0.23)$ & $0.37(0.22-0.37)$ \\
\hline & Social Anxiety 7 & $0.22(0.11-0.31)$ & $0.53(0.35-0.60)$ & $0.40(0.28-0.42)$ & $0.14(0.08-0.15)$ \\
\hline \multirow[t]{4}{*}{$\mathrm{E}$} & Negative Cognition 7 & $0.26(0.22-0.27)$ & $0.13(0.06-0.15)$ & $0.16(0.09-0.17)$ & $0.24(0.17-0.25)$ \\
\hline & Negative Affect 7 & $0.16(0.10-0.17)$ & $0.15(0.11-0.16)$ & $0.04(0.02-0.07)$ & $0.12(0.06-0.14)$ \\
\hline & Fear 7 & $0.10(0.04-0.10)$ & $0.13(0.06-0.18)$ & $0.13(0.11-0.13)$ & $0.12(0.07-0.12)$ \\
\hline & Social Anxiety 7 & $0.28(0.21-0.36)$ & $0.16(0.06-0.17)$ & $0.12(0.06-0.14)$ & $0.20(0.17-0.20)$ \\
\hline
\end{tabular}

The values on diagonal in bold represent the proportion of cross-time-within-trait co-variation attributable to additive genetics (A), shared environment (C) and to non-shared environment (E). The values on the off diagonal represent the proportions of cross-time-cross-trait co-variation for additive genetics (A), shared environment (C), and non-shared environment (E) 
relationship between negative cognition and negative affect. Not only the longitudinal cross-trait correlation for these scales was high, but also most of their longitudinal co-variation was explained by genetic factors. A possible explanation of why these two traits were more related longitudinally than other anxiety-related behaviours could lie in their phenotypic and genetic associations. The items contained within our negative cognition and negative affect scales, most closely reflect the core emotional components shared by both anxiety and depressive disorders (Clark and Watson 1991), which have also been shown to share considerable genetic influence (Hettema et al. 2006; Kendler et al. 2007; Lau and Eley 2006, 2008).Thus this overlap reflects the broader genetic overlap seen amongst anxiety and depression related traits.

The results also highlight the importance of environmental influences. Within the current analyses, several heterotypic relationships were found to be substantially explained by shared environmental factors, particularly the links between fear and negative affect. This was in accord with the results presented in cross-sectional data (Hallett et al. 2009) and resembled the relationship between fear and separation anxiety in younger children (Goldsmith and Lemery 2000). Transient nature and infinite number of environments makes finding good environmental candidates particularly difficult. However, combination of what has been reported in the current study and findings from developmental psychology presents several tantalising candidates. Research in developmental psychology suggests that environments such as parental control, parental perception of fear and parental affect, are potential causes of child's high levels of fear and negative affect. For example, adolescents' difficulties in establishing autonomy from their parent has been linked to later expression of depressed affect (Allen et al. 1994). Whereas, studies investigating the relationship between fear and attentional biases have found that negative information about a stimulus increased anxiety levels toward that stimulus (Field et al. 2001; Muris et al. 2000; Rachman 1977). Such negative information could be conveyed by parents. In terms of parental affect, mother's depression has been shown to be associated with depressionrelated negative affect in their offspring (Garber and Flynn 2001). Such parental transmission, could indicate presence of gene-environment correlation (Plomin et al. 1977), which can be passive, active or evocative. Gene-environment correlation refers to the fact that exposure to environments is not random. That is, genetic composition has an effect on environments the individual finds him/herself in. Passive gene-environment correlation, for example, occurs because parents generally pass onto their children both genes and environmental experiences. Thus, as an example, offspring of anxious mothers may receive both a genetic vulnerability for the condition and the environmental effects of an anxious parenting style. Active and evocative gene-environment correlation would inflate A estimates, whereas passive gene-environment correlation would inflate C. Such effects would be of interest to future work.

Finally, it is important to understand that, although the relationship between parent and child is unquestionably influenced by common genetic factors, our results show that what makes fear and negative affect appear in a single individual seems predominantly due to shared environmental factors. That is, a genetic predisposition may be present, but the transition from negative affect at age 7 to fear at age 9 is largely due to shared environment.

\section{Limitations}

The results should be interpreted with some potential limitations in mind. First are general limitations associated with the twin method. For example, the method assumes that MZ and DZ twin pairs will be affected similarly by their environment; the equal environment assumption. The method also overlooks the issue of chorionicity, MZ differences due to twins either sharing an amniotic sack or not (for further details and a more complete discussion of these issues see: Plomin et al. 2008). As some of these effects inflate, whilst others deflate heritability, our interpretation of these findings focuses on patterns rather than specific estimates.

Second, data were collected using a parent-rated questionnaire. Single-rater reports could result in estimation biases, which could lead to underestimation of heritability and overestimation of shared environmental influences. However, our estimates of high heritability suggested that this limitation had no major effect on our estimates. Therefore, although self-report would be a useful addition, parent data appeared to be a valid predictor of the latent levels of anxiety and depression, at least at the earlier stages of development (Kendler et al. 2008c).

Finally, although the period under investigation was very narrow (7-9 years) we felt it was particularly important to concentrate on this age range, as it is a period during which many types of anxiety cease to be part of normal development, and begin to develop into clinically significant symptoms with associated levels of impairment (Kessler et al. 2005). Nevertheless, it would be interesting to extend future analyses to also cover the period of adolescence, mainly because of the reports of new genetic influences coming 'online' during that period (Kendler et al. 2008b).

\section{Conclusions}

We showed that the homotypic continuity of specific anxiety-related behaviours (ARB's) was largely due to genetic factors. In contrast, the transmission of one type 
of anxiety into another (heterotypic continuity) particularly between fear and negative affect was strongly driven by shared-environmental factors. In middle childhood the picture of key aetiological influences is somewhat mixed. Some studies report genetic factors as central to continuity of emotional problems (van der Valk et al. 2003), whereas others posit that shared environment is the key component (Schmitz et al. 1995). Given these findings, our results show that both of these factors can be central to the maintenance of anxiety related traits but their relative role will depend on homotypic or heterotypic relationships. Furthermore, it seems that genetic versus shared environmental influences will vary depending on which aspects of anxiety are measured. Consequently, our results suggest that future environmental studies should focus on the relationship between fear and negative affect, whereas future molecular genetic research should focus on the relationship between negative cognition and negative affect.

Acknowledgments We gratefully acknowledge the ongoing contribution of the parents and children in the Twins Early Development Study (TEDS). TEDS is supported by a program grant (G0500079) from the U.K. Medical Research Council. CMAH is supported by a joint Medical Research Council (MRC), and Economic and Social Research Council (ESRC) fellowship (G0802681).

Open Access This article is distributed under the terms of the Creative Commons Attribution Noncommercial License which permits any noncommercial use, distribution, and reproduction in any medium, provided the original author(s) and source are credited.

\section{References}

Allen, J. P., Hauser, S. T., Eickholt, C., Bell, K. L., \& O’Connor, T. G. (1994). Autonomy and relatedness in family interactions as predictors of expressions of negative adolescent affect. Journal of Research on Adolescence, 4(4), 535-552.

Birmaher, B., Khetarpal, S., Brent, D., Cully, M., Balach, L., Kaufman, J., et al. (1997). The Screen for Child Anxiety Related Emotional Disorders (SCARED): scale construction and psychometric characteristics. Journal of the American Academy of Child and Adolescent Psychiatry, 36(4), 545-553.

Brady, E. U., \& Kendall, P. C. (1992). Comorbidity of anxiety and depression in children and adolescents. Psychological Bulletin, 111(2), 244-255.

Caspi, A., Henry, B., McGee, R. O., Moffitt, T. E., \& Silva, P. A. (1995). Temperamental origins of child and adolescent behavior problems: from age three to age fifteen. Child Development, 66(1), 55-68.

Clark, L. A., \& Watson, D. (1991). Tripartite model of anxiety and depression: psychometric evidence and taxonomic implications. Journal of Abnormal Psychology, 100(3), 316-336.

Costello, E. J., Mustillo, S., Erkanli, A., Keeler, G., \& Angold, A. (2003). Prevalence and development of psychiatric disorders in childhood and adolescence. Archives of General Psychiatry, 60 (8), 837-844. doi:10.1001/archpsyc.60.8.837.

Eaves, L. J., \& Silberg, J. L. (2008). Developmental-genetic effects on level and change in childhood fears of twins during adolescence. The Journal of Child Psychology and Psychiatry, 49(11), 12011210. doi:10.1111/j.1469-7610.2008.01956.x.
Eley, T. C. (1997). General genes: a new theme in developmental psychopathology. Current Directions in Psychological Science, 6 (4), 90-95.

Eley, T. C., Bolton, D., O’Connor, T. G., Perrin, S., Smith, P., \& Plomin, R. (2003). A twin study of anxiety-related behaviours in pre-school children. The Journal of Child Psychology and Psychiatry, 44(7), 945-960.

Feigon, S. A., Waldman, I. D., Levy, F., \& Hay, D. A. (2001). Genetic and environmental influences on separation anxiety disorder symptoms and their moderation by age and sex. Behavioral Genetics, 31(5), 403-411.

Ferdinand, R. F., Dieleman, G., Ormel, J., \& Verhulst, F. C. (2007). Homotypic versus heterotypic continuity of anxiety symptoms in young adolescents: evidence for distinctions between DSM-IV subtypes. Journal of Abnormal Child Psychology, 35(3), 325333. doi:10.1007/s10802-006-9093-0.

Field, A. P., Argyris, N. G., \& Knowles, K. A. (2001). Who's afraid of the big bad wolf: a prospective paradigm to test Rachman's indirect pathways in children. Behaviour Research and Therapy, 39(11), 1259-1276.

Fombonne, E., Wostear, G., Cooper, V., Harrington, R., \& Rutter, M. (2001). The Maudsley long-term follow-up of child and adolescent depression. 1. Psychiatric outcomes in adulthood. The British Journal of Psychiatry, 179, 210-217.

Ford, T., Goodman, R., \& Meltzer, H. (2003). The British Child and Adolescent Mental Health Survey 1999: the prevalence of DSMIV disorders. Journal of the American Academy of Child and Adolescent Psychiatry, 42(10), 1203-1211. doi:10.1097/01. chi.0000081820.25107.ae.

Garber, J., \& Flynn, C. (2001). Predictors of depressive cognitions in young adolescents. Cognitive Therapy and Research, 25(4), 353376. doi:10.1023/A:1005530402239.

Gladstone, G. L., Parker, G. B., Mitchell, P. B., Wilhelm, K. A., \& Malhi, G. S. (2005). Relationship between self-reported childhood behavioral inhibition and lifetime anxiety disorders in a clinical sample. Depression and Anxiety, 22(3), 103-113. doi:10.1002/da.20082.

Goldsmith, H. H., \& Lemery, K. S. (2000). Linking temperamental fearfulness and anxiety symptoms: a behavior-genetic perspective. Biological Psychiatry, 48(12), 1199-1209.

Gregory, A. M., \& Eley, T. C. (2007). Genetic influences on anxiety in children: what we've learned and where we're heading. Clinical Child and Family Psychology Review, 10(3), 199-212. doi:10.1007/s10567-007-0022-8.

Gregory, A. M., Caspi, A., Moffitt, T. E., Koenen, K., Eley, T. C., \& Poulton, R. (2007). Juvenile mental health histories of adults with anxiety disorders. The American Journal of Psychiatry, 164(2), 301-308. doi:10.1176/appi.ajp.164.2.301.

Hallett, V., Ronald, A., Rijsdijk, F., \& Eley, T. C. (2009). Phenotypic and genetic differentiation of anxiety-related behaviors in middle childhood. Depression and Anxiety, 26(4), 316-324. doi:10.1002/ da.20539.

Hettema, J. M., Neale, M. C., Myers, J. M., Prescott, C. A., \& Kendler, K. S. (2006). A population-based twin study of the relationship between neuroticism and internalizing disorders. The American Journal of Psychiatry, 163(5), 857-864. doi:10.1176/ appi.ajp.163.5.857.

Kendler, K. S., Gardner, C. O., Gatz, M., \& Pedersen, N. L. (2007). The sources of co-morbidity between major depression and generalized anxiety disorder in a Swedish national twin sample. Psychological Medicine, 37(3), 453-462. doi:10.1017/ S0033291706009135.

Kendler, K. S., Gardner, C. O., Annas, P., \& Lichtenstein, P. (2008a). The development of fears from early adolescence to young adulthood: a multivariate study. Psychological Medicine, 38(12), 1759-1769. doi:10.1017/S0033291708002936. 
Kendler, K. S., Gardner, C. O., Annas, P., Neale, M. C., Eaves, L. J., \& Lichtenstein, P. (2008b). A longitudinal twin study of fears from middle childhood to early adulthood: evidence for a developmentally dynamic genome. Archives of General Psychiatry, 65 (4), 421-429. doi:10.1001/archpsyc.65.4.421.

Kendler, K. S., Gardner, C. O., \& Lichtenstein, P. (2008c). A developmental twin study of symptoms of anxiety and depression: evidence for genetic innovation and attenuation. Psychological Medicine, 38(11), 1567-1575. doi:10.1017/ S003329170800384X.

Kessler, R. C., Berglund, P., Demler, O., Jin, R., Merikangas, K. R., \& Walters, E. E. (2005). Lifetime prevalence and age-of-onset distributions of DSM-IV Disorders in the National Comorbidity Survey Replication. Archives of General Psychiatry, 62(6), 593602. doi:10.1001/archpsyc.62.6.593.

Kovas, Y., Haworth, C. M. A., Dale, P. S., \& Plomin, R. (2007). The genetic and environmental origins of learning abilities and disabilities in the early school years 7921. Monographs of the Society for Research in Child Development, 72, 1-144.

Last, C. G., Perrin, S., Hersen, M., \& Kazdin, A. E. (1996). A prospective study of childhood anxiety disorders. Journal of the American Academy of Child and Adolescent Psychiatry, 35(11), 1502-1510.

Last, C. G., Hansen, C., \& Franco, N. (1997). Anxious children in adulthood: a prospective study of adjustment. Journal of the American Academy of Child and Adolescent Psychiatry, 36(5), $645-652$.

Lau, J. Y., \& Eley, T. C. (2006). Changes in genetic and environmental influences on depressive symptoms across adolescence and young adulthood. The British Journal of Psychiatry, 189(5), 422-427. doi:10.1192/bjp.bp.105.018721.

Lau, J. Y., \& Eley, T. C. (2008). Attributional style as a risk marker of genetic effects for adolescent depressive symptoms. Journal of Abnormal Psychology, 117(4), 849-859.

Muris, P., Merckelbach, H., Gadet, B., \& Moulaert, V. (2000). Fears, worries, and scary dreams in 4- to 12-year-old children: their content, developmental pattern, and origins. Journal of Clinical Child and Adolescent Psychology, 29(1), 43-52.

Neale, M. C. (1997). Mx: Statistical Modeling (Version 1.1). Box 710 MCV, Richmond, VA 23298: Department of Psychiatry.

Neale, M. C., \& Cardon, L. R. (1992). Methodology for genetic studies of twins and families. Dordrecht: Kluwer Academic Publications.

Oliver, B., \& Plomin, R. (2007). Twins Early Development Study (TEDS): a multivariate, longitudinal genetic investigation of language, cognition and behaviour problems from childhood through adolescence. Twin Research and Human Genetics, 10(1), 96-105.
Otto, M. W., Pollack, M. H., Maki, K. M., Gould, R. A., Worthington, J. J., 3rd, Smoller, J. W., et al. (2001). Childhood history of anxiety disorders among adults with social phobia: rates, correlates, and comparisons with patients with panic disorder. Depression and Anxiety, 14(4), 209-213. doi:10.1002/da.1068.

Pine, D. S. (2007). Editorial: answering questions in developmental science: separating the wheat from the chaff. The Journal of Child Psychology and Psychiatry, 48(12), 1157-1159. doi:10.1111/j.1469-7610.2007.01844.x.

Plomin, R., \& Kovas, Y. (2005). Generalist genes and learning disabilities. Psychological Bulletin, 131, 592-617.

Plomin, R., DeFries, J. C., \& Loehlin, J. C. (1977). Genotypeenvironment interaction and correlation in the analysis of human behaviour. Psychological Bulletin, 85, 309-322.

Plomin, R., DeFries, J. C., McClearn, G. E., \& McGuffin, P. (2008). Behavioral genetics (vol. 5th). New York: Worth Publishers.

Price, T. S., Freeman, B., Craig, I., Petrill, S. A., Ebersole, L., \& Plomin, R. (2000). Infant zygosity can be assigned by parental report questionnaire data. Twin Research, 3(3), 129-133.

Rachman, S. (1977). The conditioning theory of fear-acquisition: a critical examination. Behaviour Research and Therapy, 15(5), $375-387$.

Robinson, J. L., Reznick, J. S., Kagan, J., \& Corley, R. (1992). The heritability of inhibited and uninhibited behavior: a twin study, vol 28. Washington, DC: ETATS-UNIS: American Psychological Association.

Rosenbaum, J. F., Biederman, J., Bolduc-Murphy, E. A., Faraone, S. V., Chaloff, J., Hirshfeld, D. R., et al. (1993). Behavioral inhibition in childhood: a risk factor for anxiety disorders. Harvard Review of Psychiatry, 1(1), 2-16.

Schmitz, S., Fulker, D. W., \& Mrazek, D. A. (1995). Problem behavior in early and middle childhood: an initial behavior genetic analysis. Journal of Child Psychology and Psychiatry and Allied Disciplines, 36(8), 1443-1458.

Spence, S. H. (1997). Structure of anxiety symptoms among children: a confirmatory factor-analytic study. Journal of Abnormal Psychology, 106(2), 280-297.

Spence, S. H., Barrett, P. M., \& Turner, C. M. (2003). Psychometric properties of the Spence Children's Anxiety Scale with young adolescents. Journal of Anxiety Disorders, 17(6), 605-625.

van der Valk, J. C., van den Oord, E., Verhulst, F. C., \& Boomsma, D. I. (2003). Genetic and environmental contributions to stability and change in children's internalizing and externalizing problems. Journal of the American Academy of Child and Adolescent Psychiatry, 42(10), 1212-1220. doi:10.1097/01. chi.0000081824.25107.bb. 